\title{
On a nonlinear fractional differential equation on partially ordered metric spaces
}

\author{
Dumitru Baleanu ${ }^{1,2,3^{*}}$, Hakimeh Mohammadi ${ }^{4}$ and Shahram Rezapour ${ }^{4}$
}

\section{"Correspondence:}

dumitru@cankaya.edu.tr

'Department of Mathematics, Cankaya University, Ogretmenler Cad. 14 06530, Balgat, Ankara, Turkey

${ }^{2}$ Institute of Space Sciences, Magurele-Bucharest, Romania Full list of author information is available at the end of the article

\begin{abstract}
In this paper, by using a fixed point result on ordered metric spaces, we prove the existence and uniqueness of a solution of the nonlinear fractional differential equation $D^{\alpha} u(t)=f(t, u(t))(t \in I=[0, T], 0<\alpha<1)$ via the periodic boundary condition $u(0)=0$, where $T>0$ and $f: l \times \mathbb{R} \rightarrow \mathbb{R}$ is a continuous increasing function and ${ }^{c} D^{\alpha}$ denotes the Caputo fractional derivative of order $\alpha$. Also, we solve it by using the anti-periodic boundary conditions $u(0)+u(T)=0$ with $u(0) \leq 0$ and $u(0)+\mu u(T)=0$ with $u(0) \leq 0$ and $\mu>0$ separately.
\end{abstract}

\section{Introduction}

Fractional calculus started to be used intensively as an important tool in several branches of science and engineering (see, for example, [1-20] and the references therein). This type of calculus has an important impact in describing the dynamics of complex phenomena $[1-4,17]$. During the last few years, some new experimental confirmations have appeared in the literature in addition to the ones already established in chemistry, engineering, biology, physics, etc. As a result, the fractional differential equations were investigated intensively during the last few years. A special attention was devoted to the solvability of linear initial fractional differential equations on terms of special functions. On the other hand, the fixed point theory has wide applications in several fields (see Ref. [21] and the references therein) and it is continuously developing. Having these above mentioned things in mind, in this manuscript we have the main aim to prove the existence and uniqueness of a nonlinear fractional differential equation of Caputo type with the help of results obtained within ordered metric spaces. The paper is organized as follows. After the introductory part, in the second section, we present some of the basic tools needed in the rest of the manuscript. The third section is devoted to the main result as well as to the illustrative examples. Finally, the manuscript ends with our conclusions.

\section{Basic tools}

Recall that for a continuous function $f:[0, \infty) \rightarrow \mathbb{R}$, the Caputo derivative of fractional order $\alpha$ is defined as follows.

Definition 2.1 The Caputo fractional derivative of order $\alpha$ for a continuous function $f$ is defined by

$$
{ }^{c} D^{\alpha} f(t)=\frac{1}{\Gamma(n-\alpha)} \int_{0}^{t} \frac{f^{(n)}(s)}{(t-s)^{\alpha-n+1}} d s, \quad n=[\alpha]+1 .
$$

๑ 2013 Baleanu et al.; licensee Springer. This is an Open Access article distributed under the terms of the Creative Commons Attribution License (http://creativecommons.org/licenses/by/2.0), which permits unrestricted use, distribution, and reproduction in any medium, provided the original work is properly cited. 
Definition 2.2 The Riemann-Liouville fractional integral of order $\alpha$ is defined by

$$
I^{\alpha} f(t)=\frac{1}{\Gamma(\alpha)} \int_{0}^{t} \frac{f(s)}{(t-s)^{1-\alpha}} d s, \quad \alpha>0
$$

whenever the integral exists (see, for example, $[2,3]$ and $[18]$ ).

Theorem 2.1 Let $(M, \preceq, d)$ be a complete ordered metric space, and let $f$ be an increasing self-map on $M$. Suppose that there exist $x_{0} \in M$ and $\beta \in \Omega$ such that $x_{0} \preceq f\left(x_{0}\right)$ and $d(f(x), f(y)) \leq \beta(d(x, y)) d(x, y)$ for all $x, y \in M$ with $x \geq y$. Assume that either $f$ is continuous or $M$ has the property that if $\left\{x_{n}\right\}$ is an increasing sequence and $x_{n} \rightarrow x$ for some $x \in M$, then $x_{n} \preceq x$ for all $n$. Also, suppose that for each $x, y \in M$, there exists $z \in M$ such that $z$ is comparable with $x$ and $y$. Then $f$ has a unique fixed point.

\section{Main result}

We study the existence and uniqueness of a solution for fractional differential equations with two types of boundary conditions.

\subsection{Existence results for a nonlinear fractional differential equation in ordered metric spaces}

Consider the nonlinear fractional differential equation:

$$
{ }^{c} D^{\alpha} u(t)=f(t, u(t)) \quad(t \in I=[0, T], 0<\alpha<1)
$$

via the boundary condition $u(0)=0$, where $T>0$ and $f: I \times \mathbb{R} \rightarrow \mathbb{R}$ is a continuous function. A function $\beta$ is called a lower solution of the fractional boundary value problem whenever $\beta \in C[0, T]$ and $\beta$ satisfies ${ }^{c} D^{\alpha} \beta(t) \leq f(t, \beta(t))$ and $\beta(0) \leq 0$. Let $\Omega$ denote the class of the functions $\beta:[0, \infty) \rightarrow[0,1)$ satisfying the condition $\beta\left(t_{n}\right) \rightarrow 1$ implies $t_{n} \rightarrow 0$. Also, let $\Phi$ denote the class of increasing functions $\phi:[0, \infty) \rightarrow[0, \infty)$ such that $\phi(x)<x$ for all $x>0$ and $\frac{\phi(x)}{x} \in \Omega$. By using the following result of [21], we study the existence of solution for the above nonlinear fractional differential equation. One can compare it with the relation (6) in [13]. This nonlinear fractional differential equation has been solved for $3<\alpha \leq 4$ and different boundary value conditions [22]. Also, our technique in this section was used in [23] for solving another nonlinear fractional differential equation.

Lemma 3.1 Suppose that $0<\alpha<1, t \in I=[0, T]$ and $T>0$. Then the problem ${ }^{c} D^{\alpha} u(t)=$ $f(t, u(t))$ with the boundary value condition $u(0)=0$ is equivalent to the fractional integral equation

$$
u(t)=\int_{0}^{T} G(t, s) f(s, u(s)) d s
$$

where

$$
G(t, s)= \begin{cases}\frac{(t-s)^{\alpha-1}}{\Gamma(\alpha)}, & 0 \leq s \leq t \leq T, \\ 0, & 0 \leq t \leq s \leq T .\end{cases}
$$


Proof From ${ }^{c} D^{\alpha} u(t)=f(t, u(t)), 0<\alpha<1$ and boundary conditions, one can see that $u(t)-$ $u(0)=I^{\alpha} f(t, u(t))$. By the definition of the fractional integral, we get

$$
u(t)-u(0)=\int_{0}^{t} \frac{(t-s)^{\alpha-1}}{\Gamma(\alpha)} f(s, u(s)) d s
$$

Thus,

$$
\begin{aligned}
u(t) & =\int_{0}^{t} \frac{(t-s)^{\alpha-1}}{\Gamma(\alpha)} f(s, u(s)) d s \\
& =\int_{0}^{t} \frac{(t-s)^{\alpha-1}}{\Gamma(\alpha)} f(s, u(s)) d s+\int_{t}^{T} 0 f(s, u(s)) d s \\
& =\int_{0}^{T} G(t, s) f(s, u(s)) d s .
\end{aligned}
$$

This completes the proof.

Now, we are ready to state and prove our main result.

Theorem 3.2 Consider the nonlinear fractional differential equation ${ }^{c} D^{\alpha} u(t)=f(t, u(t))$ $(t \in I=[0, T], 0<\alpha<1)$ via the boundary condition $u(0)=0$, where $T>0, \phi \in \Phi, f$ : $I \times \mathbb{R} \rightarrow \mathbb{R}$ is an increasing continuous function and $0 \leq f(t, y)-f(t, x) \leq \frac{\Gamma(\alpha+1)}{T^{\alpha}} \phi(y-x)$ for all $x, y \in \mathbb{R}$ with $x \leq y$. If there exists a lower solution for the problem, then the problem has a unique solution.

Proof Note that by using Lemma 3.1, the problem is equivalent to the integral equation $u(t)=\int_{0}^{T} G(t, s) f(s, u(s)) d s$, where

$$
G(t, s)= \begin{cases}\frac{(t-s)^{\alpha-1}}{\Gamma(\alpha)}, & 0 \leq s<t \leq T \\ 0, & 0 \leq t<s \leq T\end{cases}
$$

Define $F: C(I, \mathbb{R}) \rightarrow C(I, \mathbb{R})$ by $(F u)(t)=\int_{0}^{T} G(t, s) f(s, u(s)) d s$. Note that $u \in C(I, \mathbb{R})$ is a fixed point of $F$ if and only if $u$ is a solution of the problem. Let $M=C(I, \mathbb{R})$. Define the order $\leq$ on $M$ by $f \leq g$ if and only if $f(t) \leq g(t)$ for all $t \in I$. Then $(M, \leq, d)$ is a complete ordered metric space, where $d(f, g)=\sup _{t \in I}|f(t)-g(t)|$. It is easy to check that $F$ is an increasing self-map. Thus,

$$
\begin{aligned}
d(F u, F v) & =\sup _{t \in I}|(F u)(t)-(F v)(t)| \\
& \leq \sup _{t \in I} \int_{0}^{T} G(t, s) f(s, u(s)) d s \\
& \leq \sup _{t \in I} \int_{0}^{T} G(t, s) \frac{\Gamma(\alpha+1)}{T^{\alpha}} \phi(u(s)-v(s)) d s
\end{aligned}
$$

for all $u \geq v$. Since the function $\phi$ is increasing and $u \geq v$, we get

$$
\phi(u(s)-v(s)) \leq \phi(d(u, v))
$$


and so

$$
\begin{aligned}
d(F u, F v) & \leq \sup _{t \in I} \int_{0}^{T} G(t, s) \frac{\Gamma(\alpha+1)}{T^{\alpha}} \phi(u(s)-v(s)) d s \\
& \leq \frac{\Gamma(\alpha+1)}{T^{\alpha}} \phi(d(u, v)) \sup _{t \in I} \int_{0}^{T} G(t, s) d s \\
& \leq \phi(d(u, v)) \frac{\Gamma(\alpha+1)}{T^{\alpha}} \frac{1}{\Gamma(\alpha+1)} T^{\alpha}=\phi(d(u, v)) \\
& =\frac{\phi(d(u, v))}{d(u, v)} d(u, v)=\beta(d(u, v)) d(u, v)
\end{aligned}
$$

for all $u \succeq v$. Now, let $\beta$ be a lower solution for the problem. We show that $\beta \preceq F(\beta)$. Since $\beta$ is a lower solution for the problem, we get $I^{\alpha} D^{\alpha} \beta(t) \leq I^{\alpha} f(t, \beta(t))$ and $u(0) \leq 0$, and so

$$
\beta(t) \leq \beta(0)+\int_{0}^{t} \frac{(t-s)^{\alpha-1}}{\Gamma(\alpha)} f(s, \beta(s)) d s
$$

for all $t \in I$. Since $u(0) \leq 0$, we have $\beta(t) \leq \int_{0}^{t} \frac{(t-s)^{\alpha-1}}{\Gamma(\alpha)} f(s, \beta(s)) d s$. Thus, we obtain $\beta(t) \leq$ $F(\beta)(t)$ for all $t \in I$. Thus, $\beta \leq F(\beta)$. Note that the space $(M, \leq, d)$ has the property that if $\left\{f_{n}\right\}$ is an increasing sequence and $f_{n} \rightarrow f$ for some $f \in M$, then $f_{n} \preceq f$ for all $n$. Therefore by using Theorem $2.1, F$ has a unique fixed point.

Example 3.1 Consider the nonlinear fractional differential equation

$$
D^{\frac{1}{2}} u(t)=f(t, u(t)) \quad\left(t \in\left[0, \frac{\pi}{2}\right]\right)
$$

via the periodic boundary condition $u(0)=0$, where $\phi(t)=\frac{4}{5} t$ and the function $f: I \times \mathbb{R} \rightarrow$ $\mathbb{R}$ is defined by $f(t, u(t))=\frac{1}{9}(t+u(t))$. Note that

$$
0 \leq f(t, y(t))-f(t, x(t))=\frac{1}{4}(y(t)-x(t)) \leq \frac{\Gamma\left(\frac{1}{2}+1\right)}{\sqrt{\frac{\pi}{2}}} \phi(y(t)-x(t))
$$

for all $x, y \in C\left(\left[0, \frac{\pi}{2}\right], \mathbb{R}\right)$ with $x \leq y$. Then, by using Theorem 3.2, the existence of a lower solution for the problem provides the existence of a unique solution.

Example 3.2 Let $m \geq 1$ be given. Consider the nonlinear fractional differential equation

$$
D^{\frac{3}{4}} u(t)=f(t, u(t)) \quad(t \in[0,1])
$$

via the boundary condition $u(0)=0$, where $\phi(t)=\frac{4}{5} t$ and the function $f: I \times \mathbb{R} \rightarrow \mathbb{R}$ is defined by $f(t, u(t))=t^{m}+\frac{1}{8} u(t)$. Note that

$$
0 \leq f(t, y(t))-f(t, x(t))=\frac{1}{8}(y(t)-x(t)) \leq \frac{\Gamma\left(\frac{3}{4}+1\right)}{1^{\frac{3}{4}}} \phi(y(t)-x(t))
$$

for all $x, y \in C([0,1], \mathbb{R})$ with $x \leq y$. Then, by using Theorem 3.2, the existence of a lower solution for the problem provides the existence of a unique solution. 


\subsection{Existence result for a nonlinear fractional differential equation with the anti-periodic boundary condition}

In this section, we solve the nonlinear fractional differential equation

$$
{ }^{c} D^{\alpha} u(t)=f(t, u(t)) \quad(t \in I=[0, T], 0<\alpha<1)
$$

via the boundary condition $u(0)+u(T)=0$ with $u(0) \leq 0$, where $T>0$ and $f: I \times \mathbb{R} \rightarrow \mathbb{R}$ is an increasing continuous function and $f \leq 0$.

Lemma 3.3 Suppose that $0<\alpha<1, t \in I=[0, T]$ and $T>0$. Then the problem ${ }^{c} D^{\alpha} u(t)=$ $f(t, u(t))$ with the boundary value condition $u(0)+u(T)=0$ with $u(0) \leq 0$ is equivalent to the fractional integral equation $u(t)=\int_{0}^{T} G(t, s) f(s, u(s)) d s$, where

$$
G(t, s)= \begin{cases}\frac{2(t-s)^{\alpha-1}-(T-s)^{\alpha-1}}{2 \Gamma(\alpha)}, & 0 \leq s \leq t \leq T, \\ \frac{-(T-s)^{\alpha-1}}{2 \Gamma(\alpha)}, & 0 \leq t \leq s \leq T .\end{cases}
$$

Proof It is easy to see that $u(t)-u(0)=I^{\alpha} f(t, u(t))$. Hence,

$$
u(t)=u(0)+\int_{0}^{t} \frac{(t-s)^{\alpha-1}}{\Gamma(\alpha)} f(s, u(s)) d s
$$

and so $u(T)=u(0)+\int_{0}^{T} \frac{(T-s)^{\alpha-1}}{\Gamma(\alpha)} f(s, u(s)) d s$. Thus,

$$
\begin{aligned}
u(0) & =\int_{0}^{T} \frac{-(T-s)^{\alpha-1}}{2 \Gamma(\alpha)} f(s, u(s)) d s \\
u(t) & =\int_{0}^{T} \frac{-(T-s)^{\alpha-1}}{2 \Gamma(\alpha)} f(s, u(s)) d s+\int_{0}^{t} \frac{(t-s)^{\alpha-1}}{\Gamma(\alpha)} f(s, u(s)) d s, \\
u(t) & =\int_{0}^{t} \frac{2(t-s)^{\alpha-1}-(T-s)^{\alpha-1}}{2 \Gamma(\alpha)} f(s, u(s)) d s-\int_{t}^{T} \frac{(T-s)^{\alpha-1}}{2 \Gamma(\alpha)} f(s, u(s)) d s \\
& =\int_{0}^{T} G(t, s) f(s, u(s)) d s .
\end{aligned}
$$

This completes the proof.

Now, we present the next result.

Theorem 3.4 Consider the nonlinear fractional differential equation ${ }^{c} D^{\alpha} u(t)=f(t, u(t))$ via the boundary condition $u(0)+u(T)=0$ with $u(0) \leq 0$, where $T>0, \phi \in \Phi, f: I \times \mathbb{R} \rightarrow \mathbb{R}$ is an increasing continuous function and $f \leq 0$ and $0 \leq f(t, y)-f(t, x) \leq \frac{2 \Gamma(\alpha+1)}{T^{\alpha}} \phi(y-x)$ for all $x, y \in \mathbb{R}$ with $x \leq y$. If there exists a lower solution for the problem, then the problem has a unique solution.

Proof Note that by using Lemma 3.3, the problem is equivalent to the integral equation $u(t)=\int_{0}^{T} G(t, s) f(s, u(s)) d s$, where

$$
G(t, s)= \begin{cases}\frac{2(t-s)^{\alpha-1}-(T-s)^{\alpha-1}}{2 \Gamma(\alpha)}, & 0 \leq s \leq t \leq T, \\ \frac{-(T-s)^{\alpha-1}}{2 \Gamma(\alpha)}, & 0 \leq t \leq s \leq T .\end{cases}
$$


Define $F: C(I, \mathbb{R}) \rightarrow C(I, \mathbb{R})$ by $(F u)(t)=\int_{0}^{T} G(t, s) f(s, u(s)) d s$. Note that $u \in C(I, \mathbb{R})$ is a fixed point of $F$ if and only if $u$ is a solution of the problem. Let $M=C(I, \mathbb{R})$. Define the order $\leq$ on $M$ by $f \leq g$ if and only if $f(t) \leq g(t)$ for all $t \in I$. Then $(M, \leq, d)$ is a complete ordered metric space, where $d(f, g)=\sup _{t \in I}|f(t)-g(t)|$. It is easy to check that $F$ is an increasing self-map. Thus,

$$
\begin{aligned}
d(F u, F v) & =\sup _{t \in I}|(F u)(t)-(F v)(t)| \\
& \leq \sup _{t \in I} \int_{0}^{T} G(t, s) f(s, u(s)) d s \\
& \leq \sup _{t \in I} \int_{0}^{T} G(t, s) \frac{2 \Gamma(\alpha+1)}{T^{\alpha}} \phi(u(s)-v(s)) d s
\end{aligned}
$$

for all $u \geq v$. Since the function $\phi$ is increasing and $u \geq v$, we get $\phi(u(s)-v(s)) \leq \phi(d(u, v))$, and so

$$
\begin{aligned}
d(F u, F v) & \leq \sup _{t \in I} \int_{0}^{T} G(t, s) \frac{2 \Gamma(\alpha+1)}{T^{\alpha}} \phi(u(s)-v(s)) d s \\
& \leq \frac{2 \Gamma(\alpha+1)}{T^{\alpha}} \phi(d(u, v)) \sup _{t \in I} \int_{0}^{T} G(t, s) d s \\
& \leq \phi(d(u, v)) \frac{2 \Gamma(\alpha+1)}{T^{\alpha}} \frac{1}{2 \Gamma(\alpha+1)} T^{\alpha}=\phi(d(u, v)) \\
& =\frac{\phi(d(u, v))}{d(u, v)} d(u, v)=\beta(d(u, v)) d(u, v)
\end{aligned}
$$

for all $u \succeq v$. Now, let $\beta$ be a lower solution for the problem. We show that $\beta \preceq F(\beta)$. Since $\beta$ is a lower solution for the problem, we get $I^{\alpha} D^{\alpha} \beta(t) \leq I^{\alpha} f(t, \beta(t)), \beta(0)+\beta(T) \leq 0$ and $\beta(0) \leq 0$. Hence,

$$
\beta(t) \leq \beta(0)+\int_{0}^{t} \frac{(t-s)^{\alpha-1}}{\Gamma(\alpha)} f(s, \beta(s)) d s
$$

and so $\beta(t) \leq-\beta(T)+\int_{0}^{t} \frac{(t-s)^{\alpha-1}}{\Gamma(\alpha)} f(s, \beta(s)) d s$ for all $t \in I$. Since $-\beta(T) \leq 0, \beta(t) \leq \int_{0}^{t} \frac{(t-s)^{\alpha-1}}{\Gamma(\alpha)} \times$ $f(s, \beta(s)) d s$ and

$$
\int_{0}^{T} \frac{-(T-s)^{\alpha-1}}{2 \Gamma(\alpha)} f(s, \beta(s)) d s \geq 0 .
$$

Thus,

$$
\beta(t) \leq \int_{0}^{t} \frac{2(t-s)^{\alpha-1}}{2 \Gamma(\alpha)} f(s, \beta(s)) d s+\int_{0}^{T} \frac{-(T-s)^{\alpha-1}}{2 \Gamma(\alpha)} f(s, \beta(s)) d s,
$$

and so

$$
\begin{aligned}
\beta(t) \leq & \int_{0}^{t} \frac{2(t-s)^{\alpha-1}-(T-s)^{\alpha-1}}{2 \Gamma(\alpha)} f(s, \beta(s)) d s \\
& +\int_{t}^{T} \frac{-(T-s)^{\alpha-1}}{2 \Gamma(\alpha)} f(s, \beta(s)) d s .
\end{aligned}
$$


Hence, $\beta(t) \leq \int_{0}^{T} G(t, s) f(s, \beta(s)) d s=(F \beta)(t)$, that is, $\beta \leq F(\beta)$. Note that the space $(M, \leq$, $d$ ) has the property that if $\left\{f_{n}\right\}$ is an increasing sequence and $f_{n} \rightarrow f$ for some $f \in M$, then $f_{n} \preceq f$ for all $n$. Therefore, by using Theorem 2.1, $F$ has a unique fixed point.

\subsection{Existence result for a nonlinear fractional differential equation with another boundary condition}

In this section, we solve the nonlinear fractional differential equation

$$
{ }^{c} D^{\alpha} u(t)=f(t, u(t)) \quad(t \in I=[0, T], 0<\alpha<1)
$$

via the boundary condition $u(0)+\mu u(T)=0$ with $u(0) \leq 0$ and $\mu>0$, where $T>0$ and $f: I \times \mathbb{R} \rightarrow \mathbb{R}$ is an increasing continuous function and $f \leq 0$.

Lemma 3.5 Suppose that $0<\alpha<1, t \in I=[0, T]$ and $T>0$. Then the problem ${ }^{c} D^{\alpha} u(t)=$ $f(t, u(t))$ with the boundary value condition $u(0)+\mu u(T)=0$ with $u(0) \leq 0$ and $\mu>0$ is equivalent to the fractional integral equation $u(t)=\int_{0}^{T} G(t, s) f(s, u(s)) d s$, where

$$
G(t, s)= \begin{cases}\frac{-\mu(T-s)^{\alpha-1}}{(\mu+1) \Gamma(\alpha)}+\frac{(t-s)^{\alpha-1}}{\Gamma(\alpha)}, & 0 \leq s \leq t \leq T \\ \frac{-\mu(T-s)^{\alpha-1}}{(\mu+1) \Gamma(\alpha)}, & 0 \leq t \leq s \leq T\end{cases}
$$

Proof It is easy to see that $u(t)-u(0)=I^{\alpha} f(t, u(t))$, and so

$$
u(t)=u(0)+\int_{0}^{t} \frac{(t-s)^{\alpha-1}}{\Gamma(\alpha)} f(s, u(s)) d s
$$

Hence, $u(T)=u(0)+\int_{0}^{T} \frac{(T-s)^{\alpha-1}}{\Gamma(\alpha)} f(s, u(s)) d s$. Since $u(T)=\frac{-u(0)}{\mu}, u(0)=\frac{-\mu}{\mu+1} \int_{0}^{T} \frac{(T-s)^{\alpha-1}}{\Gamma(\alpha)} \times$ $f(s, u(s)) d s$, and so

$$
u(t)=\int_{0}^{T} \frac{-\mu(T-s)^{\alpha-1}}{(\mu+1) \Gamma(\alpha)} f(s, u(s)) d s+\int_{0}^{t} \frac{(t-s)^{\alpha-1}}{\Gamma(\alpha)} f(s, u(s)) d s
$$

Thus,

$$
\begin{aligned}
u(t)= & \int_{0}^{t} \frac{-\mu(T-s)^{\alpha-1}}{(\mu+1) \Gamma(\alpha)}+\frac{(t-s)^{\alpha-1}}{\Gamma(\alpha)} f(s, u(s)) d s \\
& +\int_{t}^{T} \frac{-\mu(T-s)^{\alpha-1}}{(\mu+1) \Gamma(\alpha)} f(s, u(s)) d s \\
= & \int_{0}^{T} G(t, s) f(s, u(s)) d s .
\end{aligned}
$$

This completes the proof.

Now, we give the following result.

Theorem 3.6 Consider the nonlinear fractional differential equation ${ }^{c} D^{\alpha} u(t)=f(t, u(t))$ via the boundary condition $u(0)+\mu u(T)=0$ with $u(0) \leq 0$ and $\mu>0$, where $T>0, \phi \in \Phi$, $f: I \times \mathbb{R} \rightarrow \mathbb{R}$ is an increasing continuous function and $f \leq 0$ and $0 \leq f(t, y)-f(t, x) \leq$ 
$\frac{\Gamma(\alpha)}{T^{\alpha}} \phi(y-x)$ for all $x, y \in \mathbb{R}$ with $x \leq y$. If there exists a lower solution for the problem, then the problem has a unique solution.

Proof Note that by using Lemma 3.5, the problem is equivalent to the integral equation $u(t)=\int_{0}^{T} G(t, s) f(s, u(s)) d s$, where

$$
G(t, s)= \begin{cases}\frac{-\mu(T-s)^{\alpha-1}}{(\mu+1) \Gamma(\alpha)}+\frac{(t-s)^{\alpha-1}}{\Gamma(\alpha)}, & 0 \leq s \leq t \leq T \\ \frac{-\mu(T-s)^{\alpha-1}}{(\mu+1) \Gamma(\alpha)}, & 0 \leq t \leq s \leq T\end{cases}
$$

Define $F: C(I, \mathbb{R}) \rightarrow C(I, \mathbb{R})$ by $(F u)(t)=\int_{0}^{T} G(t, s) f(s, u(s)) d s$. Note that $u \in C(I, \mathbb{R})$ is a fixed point of $F$ if and only if $u$ is a solution of the problem. Let $M=C(I, \mathbb{R})$. Define the order $\leq$ on $M$ by $f \leq g$ if and only if $f(t) \leq g(t)$ for all $t \in I$. Then $(M, \leq, d)$ is a complete ordered metric space, where $d(f, g)=\sup _{t \in I}|f(t)-g(t)|$. It is easy to check that $F$ is an increasing self-map. Thus,

$$
\begin{aligned}
d(F u, F v) & =\sup _{t \in I}|(F u)(t)-(F v)(t)| \\
& \leq \sup _{t \in I} \int_{0}^{T} G(t, s) f(s, u(s)) d s \\
& \leq \sup _{t \in I} \int_{0}^{T} G(t, s) \frac{\Gamma(\alpha)}{T^{\alpha}} \phi(u(s)-v(s)) d s
\end{aligned}
$$

for all $u \geq v$. Since the function $\phi$ is increasing and $u \geq v$, we get $\phi(u(s)-v(s)) \leq \phi(d(u, v))$, and so

$$
\begin{aligned}
d(F u, F v) & \leq \sup _{t \in I} \int_{0}^{T} G(t, s) \frac{\Gamma(\alpha)}{T^{\alpha}} \phi(u(s)-v(s)) d s \\
& \leq \frac{\Gamma(\alpha)}{T^{\alpha}} \phi(d(u, v)) \sup _{t \in I} \int_{0}^{T} G(t, s) d s \\
& \leq \phi(d(u, v)) \frac{\Gamma(\alpha)}{T^{\alpha}} \frac{T^{\alpha}}{\Gamma(\alpha)}=\phi(d(u, v)) \\
& =\frac{\phi(d(u, v))}{d(u, v)} d(u, v)=\beta(d(u, v)) d(u, v)
\end{aligned}
$$

for all $u \succeq v$. Now, let $\beta$ be a lower solution for the problem. We show that $\beta \preceq F(\beta)$. Since $\beta$ is a lower solution for the problem, we get $I^{\alpha} D^{\alpha} \beta(t) \leq I^{\alpha} f(t, \beta(t)), \beta(0)+\mu \beta(T) \leq 0$ and $\beta(0) \leq 0$. Hence, $\beta(t) \leq \beta(0)+\int_{0}^{t} \frac{(t-s)^{\alpha-1}}{\Gamma(\alpha)} f(s, \beta(s)) d s$, and so

$$
\beta(t) \leq \int_{0}^{t} \frac{(t-s)^{\alpha-1}}{\Gamma(\alpha)} f(s, \beta(s)) d s
$$

for all $t \in I$. Since $f \leq 0, \int_{0}^{T} \frac{-\mu(T-s)^{\alpha-1}}{(\mu+1) \Gamma(\alpha)} f(s, \beta(s)) d s \geq 0$. Thus,

$$
\begin{aligned}
\beta(t) \leq & \int_{0}^{t} \frac{-\mu(T-s)^{\alpha-1}}{(\mu+1) \Gamma(\alpha)}+\frac{(t-s)^{\alpha-1}}{\Gamma(\alpha)} f(s, \beta(s)) d s \\
& +\int_{t}^{T} \frac{-\mu(T-s)^{\alpha-1}}{(\mu+1) \Gamma(\alpha)} f(s, \beta(s)) d s,
\end{aligned}
$$


and so $\beta(t) \leq \int_{0}^{T} G(t, s) f(s, \beta(s)) d s=(F \beta)(t)$. Hence, $\beta \leq F(\beta)$. Note that the space $(M, \leq$, $d$ ) has the property that if $\left\{f_{n}\right\}$ is an increasing sequence and $f_{n} \rightarrow f$ for some $f \in M$, then $f_{n} \preceq f$ for all $n$. Therefore by using Theorem 2.1, $F$ has a unique fixed point.

\section{Conclusions}

In this manuscript, by making use of fixed point techniques on ordered metric spaces, the existence and uniqueness of the solution of a nonlinear fractional differential equation with periodic and anti-periodic boundary conditions were investigated. To sustain our results, two illustrative examples were analyzed in detail.

Competing interests

The authors declare that they have no competing interests.

Authors' contributions

All authors have equal contributions.

\section{Author details}

${ }^{1}$ Department of Mathematics, Cankaya University, Ogretmenler Cad. 14 06530, Balgat, Ankara, Turkey. ${ }^{2}$ Institute of Space Sciences, Magurele-Bucharest, Romania. ${ }^{3}$ Department of Chemical and Materials Engineering, Faculty of Engineering, King Abdulaziz University, Jeddah, Saudi Arabia. ${ }^{4}$ Department of Mathematics, Azarbaidjan Shahid Madani University, Azarshahr, Tabriz, Iran.

\section{Acknowledgements}

This work is partially supported by the Scientific and Technical Research Council of Turkey. Research of the second and third authors was supported by Azarbaidjan Shahid Madani University.

Received: 5 December 2012 Accepted: 5 March 2013 Published: 29 March 2013

\section{References}

1. Miller, KS, Ross, B: An Introduction to the Fractional Calculus and Fractional Differential Equation. Wiley, New York (1993)

2. Kilbas, AA, Srivastava, HM, Trujillo, JJ: Theory and Applications of Fractional Differential Equations. North-Holland Mathematics Studies, vol. 204. Elsevier, Amsterdam (2006)

3. Podlubny, I: Fractional Differential Equations. Academic Press, San Diego (1999)

4. Samko, SG, Kilbas, AA, Marichev, Ol: Fractional Integral and Derivative, Theory and Applications. Gordon \& Breach, New York (1993)

5. Baleanu, D: Fractional variational principles in action. Phys. Scr. 2009(T136), Article ID 014006 (2009)

6. Tenreiro, JA: Fractional derivatives: probability interpretation and frequency response of rational approximations. Commun. Nonlinear Sci. Numer. Simul. 14, 3492-3497 (2009)

7. Delbosco, D, Rodino, L: Existence and uniqueness for a nonlinear fractional differential equation. J. Math. Anal. Appl. 204, 609-925 (1996)

8. Zhang, S: The existence of a positive solution for nonlinear fractional differential equation. J. Math. Anal. Appl. 252, 804-812 (2000)

9. Zhang, S: Existence of positive solutions for some class of nonlinear fractional equation. J. Math. Anal. Appl. 278, $136-148(2003)$

10. Hashim, I, Abdulaziz, O, Momani, S: Homotopy analysis method for fractional initial value problems. Commun Nonlinear Sci. Numer. Simul. 14, 674-684 (2009)

11. Al-Mdallal, M, Syam, MI, Anwar, MN: A collocation-shooting method for solving fractional boundary value problems. Commun. Nonlinear Sci. Numer. Simul. 15, 3814-3822 (2010)

12. Jafari, $\mathrm{H}$, Daftardar-Gejji, $\mathrm{V}$ : Positive solution of nonlinear fractional boundary value problems using a domain decomposition method. J. Appl. Math. Comput. 180, 700-706 (2006)

13. Ahmad, B, Nieto, JJ: Existence of solutions for nonlocal boundary value problems of higher-order nonlinear fractional differential equations. Abstr. Appl. Anal. 2009, Article ID 494720 (2009)

14. Nieto, JJ: Maximum principles for fractional differential equations derived from Mittag-Leffler functions. Appl. Math. Lett. 23, 1248-1251 (2010)

15. Belmekki, M, Nieto, JJ, Rodriguez-Lopez, R: Existence of periodic solution for a nonlinear fractional differential equation. Bound. Value Probl. 2009, Article ID 324561 (2009)

16. Qiu, T, Bai, Z: Existence of positive solution for singular fractional equations. Electron. J. Differ. Equ. 146, 1-9 (2008)

17. Baleanu, D, Diethelm, K, Scalas, E, Trujillo, JJ: Fractional Calculus Models and Numerical Methods. Series on Complexity, Nonlinearity and Chaos. World Scientific, Singapore (2012)

18. Baleanu, D, Mustafa, OG: On the global existence of solutions to a class of fractional differential equations. Comput. Math. Appl. 59(5), 1835-1841 (2010)

19. Agarwal, RP, Lakshmikantam, V, Nieto, JJ: On the concept of solution for fractional differential equations with uncertainty. Nonlinear Anal. 72, 2859-2862 (2010)

20. Zhao, Y, Sun, Sh, Han, Z: The existence of multiple positive solutions for boundary value problems of nonlinear fractional differential equations. Commun. Nonlinear Sci. Numer. Simul. 16, 2086-2097 (2011) 
21. Amini-Harandi, A, Emami, H: A fixed point theorem for contraction type maps in partially ordered metric spaces and application to ordinary differential equations. Nonlinear Anal. 72, 2238-2242 (2010)

22. Cabrera, IJ, Harjani, J, Sadarangani, KB: Existence and uniqueness of positive solutions for a singular fractional three-point boundary value problem. Abstr. Appl. Anal. 2012, Article ID 803417 (2012)

23. Zhou, $\mathrm{X}, \mathrm{Wu}, \mathrm{W}, \mathrm{Ma}, \mathrm{H}$ : A contraction fixed point theorem in partially ordered metric spaces and application to fractional differential equations. Abstr. Appl. Anal. 2012, Article ID 856302 (2012)

doi:10.1186/1687-1847-2013-83

Cite this article as: Baleanu et al.: On a nonlinear fractional differential equation on partially ordered metric spaces. Advances in Difference Equations 2013 2013:83.

Submit your manuscript to a SpringerOpen ${ }^{\circ}$ journal and benefit from:

- Convenient online submission

- Rigorous peer review

- Immediate publication on acceptance

- Open access: articles freely available online

- High visibility within the field

- Retaining the copyright to your article 